ESAIM: PROCEEDINGS, December 2012, Vol. 38, p. 202-219

F. Coquel, M. Gutnic, P. Helluy, F. Lagoutière, C. Rohde, N. Seguin, Editors

\title{
A MULTISCALE PROBLEM IN THERMAL SCIENCE
}

\author{
Fabien Casenave ${ }^{1,2}$, Mohamed Ghattassi ${ }^{3}$ and Rémi Joubaud ${ }^{1,4}$
}

\begin{abstract}
We consider a multiscale heat problem in civil aviation: determine the temperature field in a plane in flying conditions, with air conditioning. Ventilated electronic components in the bay bring a heat source, introducing a second scale in the problem. First, we present three levels of modelling for the physical phenomena, which are applied to the two sub-problems: the plane and the electronic component. Then, having reduced the complexity of the problem to a linear non-symmetric coercive PDE, we will use the reduced basis method for the electronic component problem.

Résumé. Nous considérons un problème multi-échelle d'aérothermie en aviation civile. Nous souhaitons déterminer le champ de température dans un avion en conditions de vol, avec présence d'une climatisation. Des composants électroniques ventilés sont présents dans la soute, et constituent une source de chaleur, introduisant une deuxième échelle dans notre problème. Dans un premier temps, nous présentons trois niveaux de modélisation pour le phénomène d'aérothermie, que nous appliquerons aux deux sous-problèmes: l'avion et le composant électronique. Ensuite, nous appliquons la méthode des bases réduites au problème du composant électronique, en considérant des simplifications de modélisation amenant à la résolution numérique d'une EDP elliptique linéaire coercive non-symétrique.
\end{abstract}

\section{INTRODUCTION}

In the civil aircraft industry, one of the main stakes is fuel efficiency. The use of composite materials, to replace aluminum alloys, enables the manufacturers to lighten the plane while keeping the required mechanical properties. However, these materials present lower thermal conductivity, leading to new air conditioning problems. The goal of the present work is to develop fast tools to compute the temperature in an aircraft cabin in flying conditions, with presence of heat sources: mainly the electronic components.

A closer look will be taken at two problems:

- the passenger comfort in which case the output of interest is the temperature in the cabin;

- the equipment failure in which case the output of interest is the maximum of the temperature in the electronic components.

After presenting three levels of modelling for the physical phenomena, we present numerical simulations of the model providing the best trade-off between physical realism and computational accuracy. Then, a reduced basis approach will be developed for the electronic component problem to further speed up the computations.

1 Université Paris Est, CERMICS, Ecole des Ponts ParisTech, 6 \& 8 Av. Pascal, 77455 Marne-la-Vallée, France

2 EADS France, Bâtiment Campus Engineering - Service IW/SE/MA, BP 90112 - 31703 BLAGNAC Cedex

3 Institut Élie Cartan, Université Henri Poincaré Nancy 1, F-54506 Vandoeuvre-lès-Nancy Cedex

${ }^{4}$ ANDRA, DRD/EAP, Parc de la croix blanche, 1,7 rue Jean Monnet, 92298 Châtenay-Malabry Cedex, France

(C) EDP Sciences, SMAI 2012 


\section{Physical MODELLing}

In both cabin and equipment problems, the flow as well as the temperature have to be computed. We first introduce three models of increasing complexity to solve this physical problem. The standard continuum model for natural convection phenomenon is the compressible Navier-Stokes system (CNS). This model present both theoretical and numerical difficulties in the sense that the equations of conservation are strongly coupled and nonlinear. We choose to consider a hierarchy of simplifications of (CNS).

\subsection{A hierarchy of models}

Consider a bounded domain $\Omega \subset \mathbb{R}^{2}$ representing the cabin or the electronic components. The domain $\Omega$ is split in two parts:

$$
\Omega=\Omega_{\text {solid }} \cup \Omega_{\text {air }},
$$

where $\Omega_{\text {solid }}$ stands for solid structures in the cabin or in the electric component. Thus, the velocity field is considered as non-zero on $\Omega \backslash \Omega_{\text {solid }}$ and extended by 0 on $\Omega_{\text {solid }}$. As a first simplification, we will consider the Boussinesq equations, neglecting density variations except in the body force so that the fluid is divergence free. The coupling between the velocity and temperature fields appears in the body force terms in the equations of the fluid and the advective term in the heat equation. This model expresses conservation of momentum and mass of the fluid coupled to the heat equation. The unsteady equations of conservations reads, for a time $t_{\mathrm{S}}>0$, for all $t \in\left[0, t_{\mathrm{S}}\right]$ :

$$
\left\{\begin{aligned}
\rho_{0}\left(\frac{\partial \boldsymbol{u}}{\partial t}+\boldsymbol{u} \cdot \nabla \boldsymbol{u}\right) & =\rho_{0}\left(1-\frac{T-T_{0}}{T_{0}}\right) \boldsymbol{g}-\nabla p+\eta \Delta \boldsymbol{u} \text { in } \Omega_{\mathrm{air}}, \\
\operatorname{div}(\boldsymbol{u}) & =0 \text { in } \Omega_{\mathrm{air}}, \\
\rho_{0} c_{p}(x)\left(\frac{\partial T}{\partial t}+\boldsymbol{u} \cdot \nabla T\right) & =\operatorname{div}(\kappa(x) \nabla T)+Q(x) \text { in } \Omega,
\end{aligned}\right.
$$

Here, $\boldsymbol{u}$ denote the velocity field, $p$ the pressure and $T$ the temperature. $T_{0}$ is a reference temperature (300 Kelvin), $\rho_{0}$ is the air density at temperature $T_{0}, \boldsymbol{g}$ the gravity constant, $\eta$ the air dynamic viscosity. Then, $c_{p}$ and $\kappa$ are space dependent discontinuous functions and represent the heat capacity and the heat conductivity of the considered medium (e.g air, aircraft structure). Eventually, $Q$ is a space depend-ant function representing a source term in the heat problem. In the following, the function $\kappa, c_{p}, Q$ have the form

$$
\kappa(x)=\sum_{i} \kappa_{i} \mathbf{1}_{\Omega_{i}}, \quad c_{p}(x)=\sum_{i} c_{p}^{i} \mathbf{1}_{\Omega_{i}}, \quad Q(x)=\sum_{i} Q_{i} \mathbf{1}_{\Omega_{i}}
$$

where indices $i$ refer to the air and different solid parts depending on the considered problem (aircraft structure, electronic component part,...).

If we assume that the variations of temperature do not modify the velocity field, we can decouple the fluid and heat problems. Moreover, we consider the fluid at steady state thereby neglecting any feedback of the temperature on the convection of the air. The conservation equations writes for a time $t_{\mathrm{S}}>0$, for all $t \in\left[0, t_{\mathrm{S}}\right]$ :

$$
\left\{\begin{aligned}
\rho_{0} \boldsymbol{u}_{\mathrm{NS}} \cdot \nabla \boldsymbol{u}_{\mathrm{NS}} & =\rho_{0} \boldsymbol{g}-\nabla p+\eta \Delta \boldsymbol{u}_{\mathrm{NS}} \text { in } \Omega_{\mathrm{air}}, \\
\operatorname{div}\left(\boldsymbol{u}_{\mathrm{NS}}\right) & =0 \text { in } \Omega_{\mathrm{air}}, \\
\rho_{0} c_{p}(x)\left(\frac{\partial T}{\partial t}+\boldsymbol{u}_{\mathrm{NS}} \cdot \nabla T\right) & =\operatorname{div}(\kappa(x) \nabla T)+Q(x) \text { in } \Omega .
\end{aligned}\right.
$$

The last level of modelling consists, as in the previous case, in taking a stationary regime and in decoupling the fluid and heat problems for the same reasons. The next simplification is to consider a basic model for the fluid equation, namely a potential flow. All the phenomena induced by the viscosity are not captured by this type 
of model (recirculation zones, boundary layers).

The conservation equations writes:

$$
\left\{\begin{aligned}
-\Delta \psi & =0 \text { in } \Omega_{\mathrm{air}} \\
\rho_{0} c_{p}(x)\left(\partial_{t} T+\boldsymbol{u}_{\mathrm{pot}} \cdot \nabla T\right) & =\operatorname{div}(\kappa(x) \nabla T)+Q(x) \text { in } \Omega
\end{aligned}\right.
$$

Here, $\boldsymbol{u}_{\text {pot }}=\nabla \psi$ is the velocity field associated with the potential $\psi$. All the three models are supplemented with initial and boundary conditions. A nice consequence for the numerical calculations is that for the two last systems (3) and (4), a single evaluation of the velocity field is required.

\subsection{Geometry and boundary conditions}

Now, we describe the different boundary conditions that we consider in the numerical experiments. Assume that the boundary of $\Omega$ is partitioned as follow:

$$
\partial \Omega=\Gamma_{\text {in }} \cup \Gamma_{\text {out }} \cup \Gamma_{\text {wall }}
$$

The portions $\Gamma_{\text {in/out }}$ represent parts of the domain where there are exchange of air (fans and evacuations) and $\Gamma_{\text {wall }}$ are solid adiabatic walls. We enforce non-penetration and no-slip boundary conditions for the fluid flow on the walls:

$$
\boldsymbol{u}=0 \text { on } \Gamma_{\text {wall }},
$$

and an inflow of air is imposed on $\Gamma_{\text {in }}$ through a Dirichlet boundary condition:

$$
\left\{\begin{aligned}
\boldsymbol{u} \cdot \boldsymbol{n} & =u_{\text {in }} \text { on } \Gamma_{\text {in }} \\
\eta(\nabla \boldsymbol{u} \cdot \boldsymbol{n}) \cdot \boldsymbol{\tau} & =0 \text { on } \Gamma_{\text {in }}
\end{aligned}\right.
$$

where $u_{\text {in }}$ is a scalar function that we will specify for the numerical calculations and $\tau$ a tangent vector to $\Gamma_{\text {in }}$. In the case of systems (1) and (3), we enforce natural boundary condition, requiring:

$$
\eta(\nabla \boldsymbol{u} \cdot \boldsymbol{n})=p \boldsymbol{n} \text { on } \Gamma_{\text {out }} .
$$

In the case of system (4), in order to have a well posed problem, we impose exact conservation of mass enforcing:

$$
\boldsymbol{u}_{\text {pot }} \cdot \boldsymbol{n}=0 \text { on } \Gamma_{\text {out }},
$$

so that the Poisson problem for the potential flow has to be solved with non-homogeneous Neumann boundary conditions. With this setting, the potential is determined up to an additive constant. For the numerical experiments we fix $\int_{\Omega_{\text {air }}} \psi=0$. This condition ensure the well-posedness of the problem.

For the temperature field, we assume Dirichlet boundary conditions in the inflow part. Since we assume that the wall are adiabatic, the boundary conditions for the temperature reads:

$$
\left\{\begin{array}{l}
T=T_{\text {in }} \text { on } \Gamma_{\text {in }}, \\
\nabla T \cdot \boldsymbol{n}=0 \text { on } \Gamma_{\text {out }} \cup \Gamma_{\text {wall }}
\end{array}\right.
$$

\subsection{Time and space discretization}

We now describe the numerical implementation used to solve the previous systems of conservation equations. We describe the numerical algorithm used to solve unsteady Navier-Stokes equations and the heat equation. We use those algorithms in an iterative process to solve the Boussinesq equations. We start by describing the Navier-Stokes solver. The discretization is based on finite elements in space and implicit Euler scheme in time. Let $\delta t$ be the time step, taken to be constant for simplicity. We denote by $t^{n}=n \delta t$ the $n$-th discrete time. 
We introduce $V_{h}\left(\Omega_{\text {air }}\right)^{2}$ and $M_{h}\left(\Omega_{\text {air }}\right)$ the finite elements spaces for velocity and pressure. We define the fluid problem at time $t^{n+1}$ for a temperature $T$ by: given $\boldsymbol{u}^{n} \in V_{h}\left(\Omega_{\text {air }}\right)^{2}$, we seek $\left(\boldsymbol{u}^{n+1}, p^{n+1}\right) \in V_{h}\left(\Omega_{\text {air }}\right)^{2} \times M_{h}\left(\Omega_{\text {air }}\right)$ such that for all $(\boldsymbol{v}, q) \in V_{h}\left(\Omega_{\text {air }}\right)^{2} \times M_{h}\left(\Omega_{\text {air }}\right)$,

$$
\text { Fluid }(n+1, T):=\left\{\begin{array}{l}
\frac{1}{\delta t} \int_{\Omega_{\mathrm{air}}} \rho_{0} \boldsymbol{u}^{n+1} \cdot \boldsymbol{v} d \boldsymbol{x}+\int_{\Omega_{\mathrm{air}}} \rho_{0}\left(\boldsymbol{u}^{n+1} \nabla \boldsymbol{u}^{n+1}\right) \cdot \boldsymbol{v} d \boldsymbol{x}+\eta \int_{\Omega_{\mathrm{air}}} \nabla \boldsymbol{u}^{n+1}: \nabla \boldsymbol{v} d \boldsymbol{x} \\
-\int_{\Omega_{\mathrm{air}}} p^{n+1} \operatorname{div}(\boldsymbol{v}) d \boldsymbol{x}=\frac{1}{\delta t} \int_{\Omega_{\mathrm{air}}} \rho_{0} \boldsymbol{u}^{n} \cdot \boldsymbol{v} d \boldsymbol{x}+\int_{\Omega_{\mathrm{air}}} \rho_{0}\left(1-\frac{T-T_{0}}{T_{0}}\right) \boldsymbol{g} \cdot \boldsymbol{v} d \boldsymbol{x}, \\
\int_{\Omega_{\mathrm{air}}} q \operatorname{div}\left(\boldsymbol{u}^{n+1}\right) d \boldsymbol{x}=0 .
\end{array}\right.
$$

Let us denote $\tilde{\boldsymbol{u}}$ the extension by zero of $\boldsymbol{u}$ on $\Omega$. We search the temperature in the same finite element space than the velocity components. We define the heat problem at time $t^{n+1}$ for a fluid velocity $\tilde{\boldsymbol{u}}$ by: given $T^{n} \in M_{h}(\Omega)$, we seek $T^{n+1} \in M_{h}(\Omega)$ such that for all $\Theta \in M_{h}(\Omega)$ :

$$
\text { Heat }(n+1, \tilde{\boldsymbol{u}}):=\left\{\begin{array}{l}
\frac{1}{\delta t} \int_{\Omega} \rho_{0} c_{p}(x) T^{n+1} \Theta d \boldsymbol{x}+\int_{\Omega} \rho_{0} c_{p}(x)\left(\tilde{\boldsymbol{u}} \cdot \nabla T^{n+1}\right) \Theta d \boldsymbol{x}+ \\
\int_{\Omega} k(x) \nabla T^{n+1} \cdot \nabla \Theta d \boldsymbol{x}=\int_{\Omega} Q(x) \Theta d \boldsymbol{x}+\frac{1}{\delta t} \int_{\Omega} \rho_{0} c_{p}(x) T^{n} \Theta d \boldsymbol{x} .
\end{array}\right.
$$

The variational formulation for steady Navier-Stokes equations and equation can be straightforwardly obtained from (10) and (11). Note that the time scheme for the system (10) is nonlinear and we resort to a Newton-Raphson algorithm to solve this nonlinear problem at each time step. In the case of the potential flow, one can resort to a variational formulation of the Poisson equation with Neumann boundary conditions in conjunction with a Lagrange multiplier to ensure that $\int_{\Omega_{\mathrm{air}}} \psi=0$. For instance, we take the inf-sup stable pair of discrete finite elements spaces $V_{h}\left(\Omega_{\text {air }}\right)^{2} \times M_{h}\left(\Omega_{\text {air }}\right)=\left(\mathbb{P}_{2}\right)^{2} \times \mathbb{P}_{1}$ for the fluid equations and $M_{h}(\Omega)=\mathbb{P}_{1}$ for the heat equation. Let us depict the iterative process allowing to solve the Boussinesq system:

Initialize with $\boldsymbol{u}_{0} \in V_{h}\left(\Omega_{\text {air }}\right)^{2}$ and $T_{0} \in M_{h}(\Omega)$;

For all $n \geq 0$,

(i) solve Fluid $\left(n+1, T^{n}\right)$ to get $\boldsymbol{u}^{n+1}$;

(ii) solve Heat $\left(n+1, \boldsymbol{u}^{n+1}\right)$ to get $T^{n+1}$;

All calculations are performed using the finite element solver FreeFEM++ (see [5]). As a matter of illustration, the computational code was tested on a simple configuration: consider a square box, with initial state uniform temperature and fluid at rest. A source term $Q$ for the heat problem is localized in the lower part of the box. As we can see in Figure 1, the temperature dependent density in the gravitational term lightens hot air and
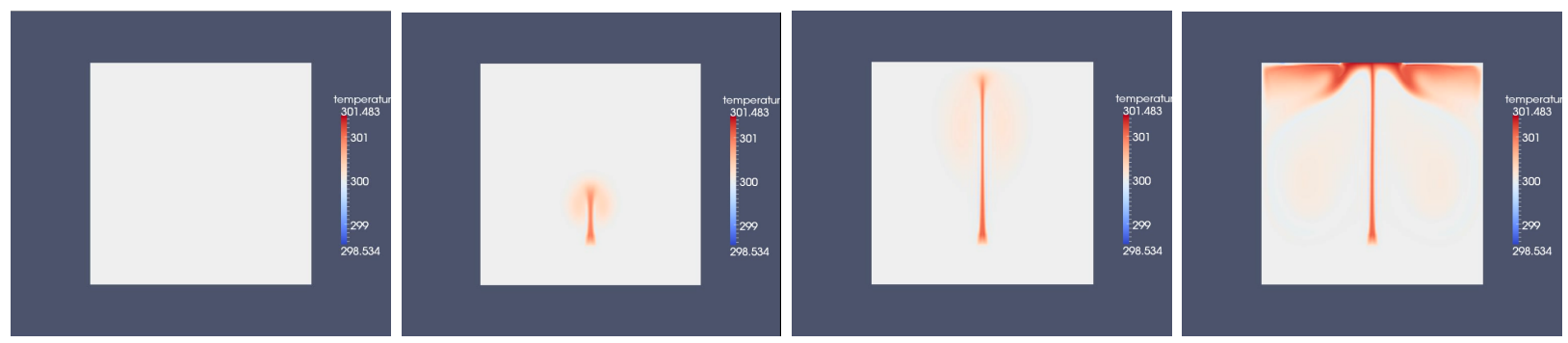

Figure 1. Temperature field at times $\mathrm{t}=0,0.5,1$ and $4 \mathrm{~s}$

weighs down cold air. This enable a nonzero velocity field to arise, and a convective dissipation of the heat. 


\subsection{Numerical results}

\subsubsection{The plane}

Consider a bidimensional cross section of a plane (see Figure 2). The upper part, the cabin, presents an inflow of cool air coming from an air conditioning system. Air is leaving the plane through a hole in the lower part, the bay, which contains the electronic components that create heat by Joule effect. A simplified geometry is considered: the goal here is to develop a methodology, not to carry out an accurate industrial simulation. We

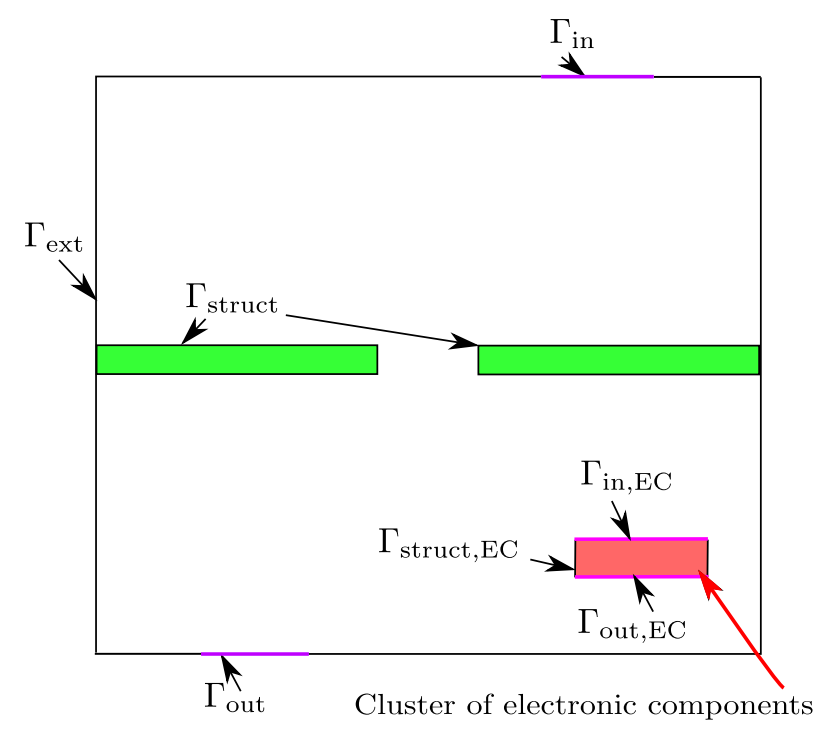

Figure 2. Geometry of the plane test case

consider that the heat produced by the components is completely brought to the bay. Therefore, we can take a constant effective surfacic source term $Q$ in the heat problem. The velocity on $\Gamma_{\text {in, EC }}$ is imposed by the fans of the components. This methodology has been applied for the three different models considered previously.

- Unsteady Boussinesq:

See Figures 3 and 4.

As expected in such physical situation, the Boussinesq solution does not reach a steady state. The convection effects induced by temperature gradients are the dominant effects, therefore the Boussinesq coupling could not be simplified. Cool air is coming from the upper part and the air in the lower part is being heated up, and the hydrostatic equilibrium is not reached at the end of the simulation.

- Steady decoupled NS/heat:

See Figure 5.

The velocity field has been computed once for all, and is assumed not to depend on the temperature. The air viscosity produces recirculation zones. Without Boussinesq effect, the cooling down is only ensured by diffusion and convection from the precomputed velocity field. These two effects are too low, and the temperature reaches very high values.

- Unsteady decoupled potential/heat:

See Figure 6.

The precomputed flow is very simple, viscosity is neglected. Recirculation zones are not obtained and therefore the cooling of the components (away from the air conditioning main stream) is even worse. Diffusion and convection are way too low, and the temperature diverges. 

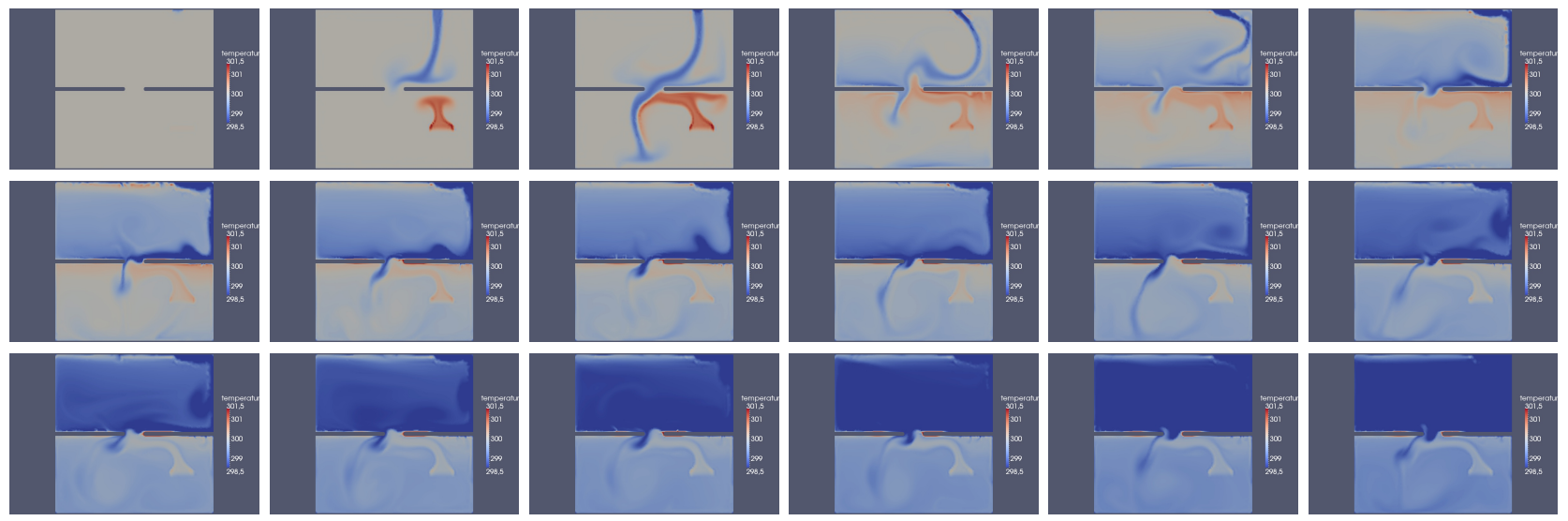

Figure 3. Temperature field in the Boussinesq model, with the cluster at the right of the bay, at times $0,100,200, \ldots 1700$
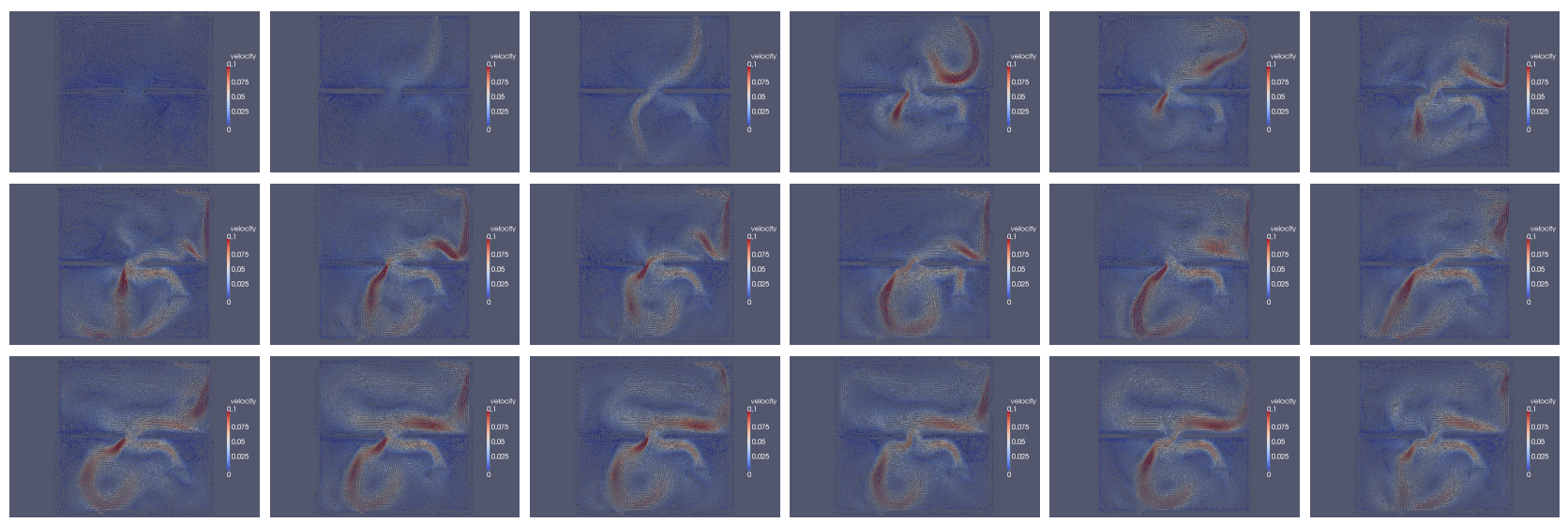

FiguRE 4. Velocity field in the Boussinesq model, with the cluster at the right of the bay, at times $0,100,200, \ldots 1700$

For this situation, only the unsteady Boussinesq can capture all the physical phenomena, and is therefore the model to be considered. In the two other models, the convection brought by the Boussinesq term is not captured, and the components are not cooled down enough. Note that there do not seem to be any steady state to reach for this problem. We simulated up to $40 \mathrm{~min}$, and the velocity and temperature fields appear to reach a periodic in time behavior.

In the present section, we simply modelled the presence of electronic components by a constant surfacic heat source term. We will now develop a model to simulate the velocity and the temperature inside the electronic component.

\subsubsection{The electronic component}

Consider a 2D section of an electronic component (see Figure 7). The green area represent the support board for the red-colored integrated circuits. The blue zone is filled with air, pushed from the bottom part by a fan, and leaving the box through the top part. The red components will heat up by Joule effect while functioning. Periodic boundary conditions are enforced at $\Gamma_{\text {per }}$. 

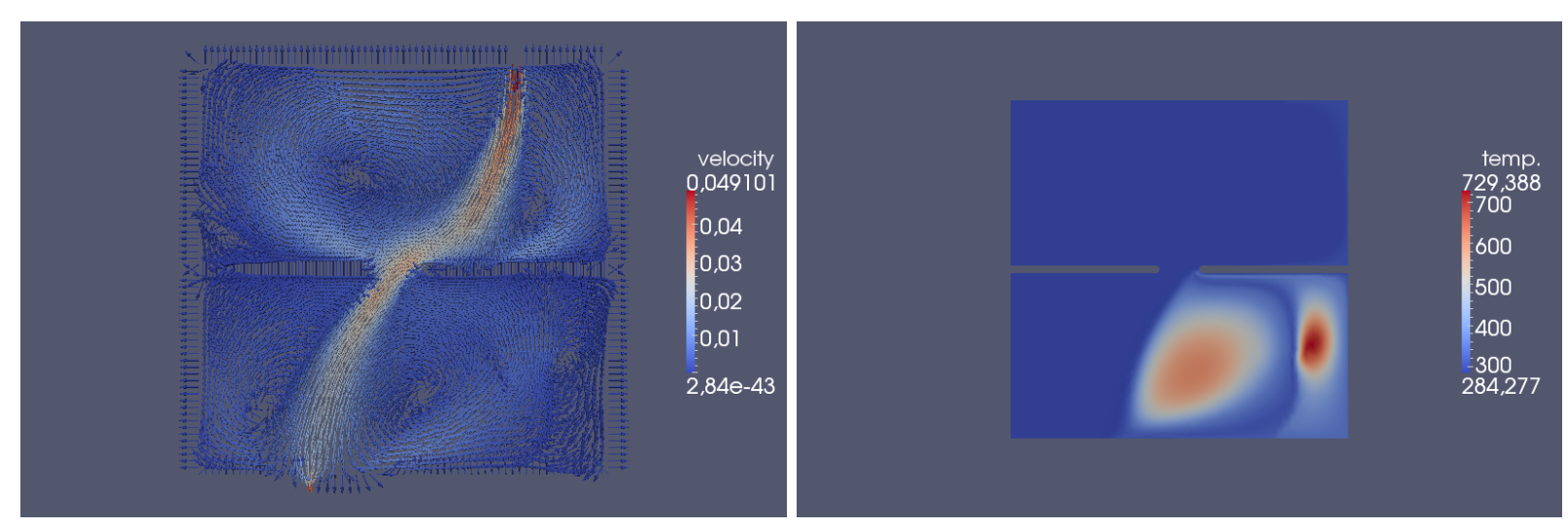

FiguRE 5. Plane test case in the decoupled NS/heat model. Velocity and temperature fields.
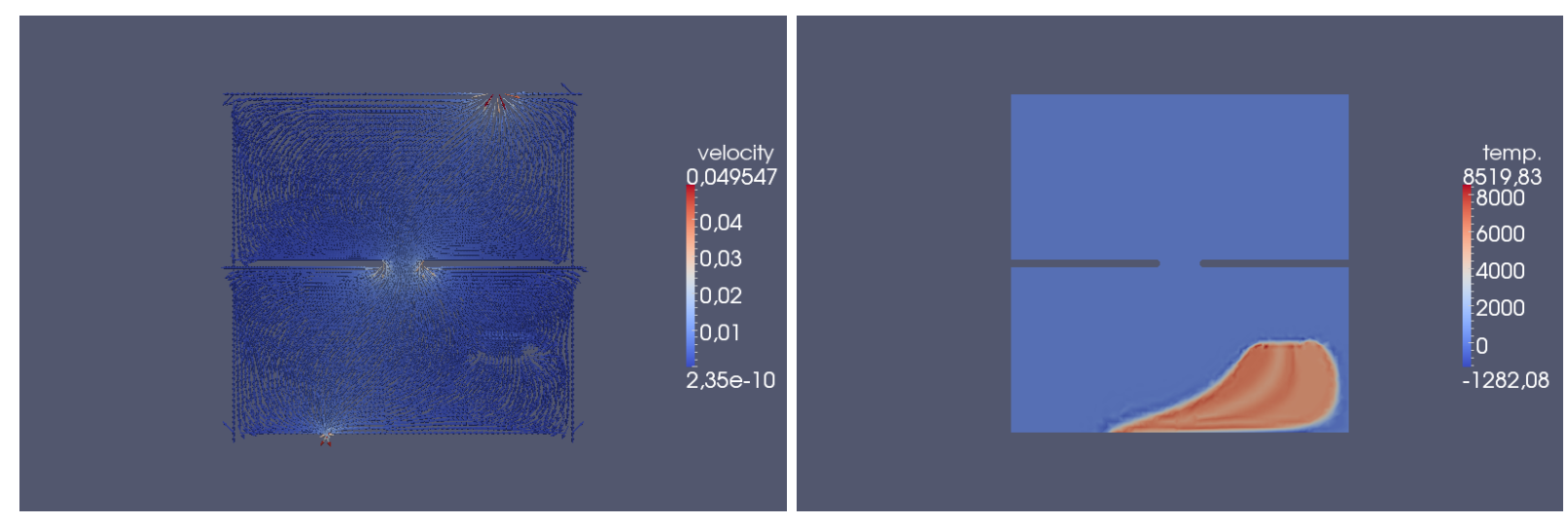

Figure 6. Plane test case in the decoupled potential/heat model. Velocity and temperature fields.

In this case, the steady decoupled incompressible Navier-Stokes / heat model gives the same result as the long time Boussinesq (see Figure 8). Actually, the Péclet number is large $\left(\approx 10^{4}\right)$, indicating that convective effects are dominant. Moreover, the flux is strongly guided inside a channel, which is different from the previous case where air was blown inside a large volume at rest. Forced convection dominates convection induced by local thermal fluxes. The unsteady decoupled potential / heat is not satisfactory: the absence of boundary layer does not allow the air close to the integrated circuits to heat up. In this case, contrary to the previous one, the convection is overestimated in the heating area.

As a conclusion, a decoupling of the fluid and the heat problems is possible. For instance, in order to determine the conductivities and heat capacities of the elements of the electronic component that minimize the temperature, a reduced basis approach can be carried out to drastically reduce the computation time in such a many queries context.

\section{A REDUCED BASIS APPROACH FOR THE ELECTRONIC COMPONENT PROBLEM}

\subsection{Review of the method}

The reduced basis $(\mathrm{RB})$ method aims at reducing the computation time in a precise context: running many times the same calculation with a small change of a set of parameters. The idea is somehow close to modal 


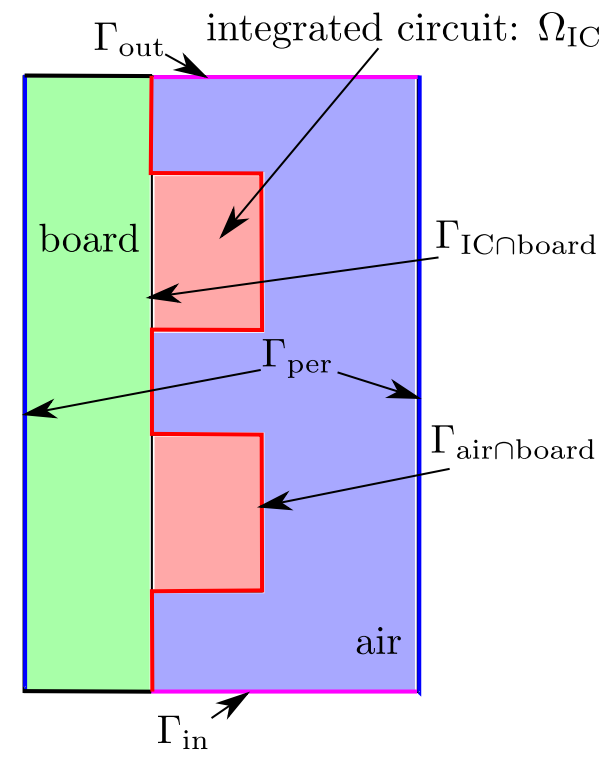

FigURE 7. Distorted geometry of the electronic component test case (the height of the component is actually 16 times larger than its width)
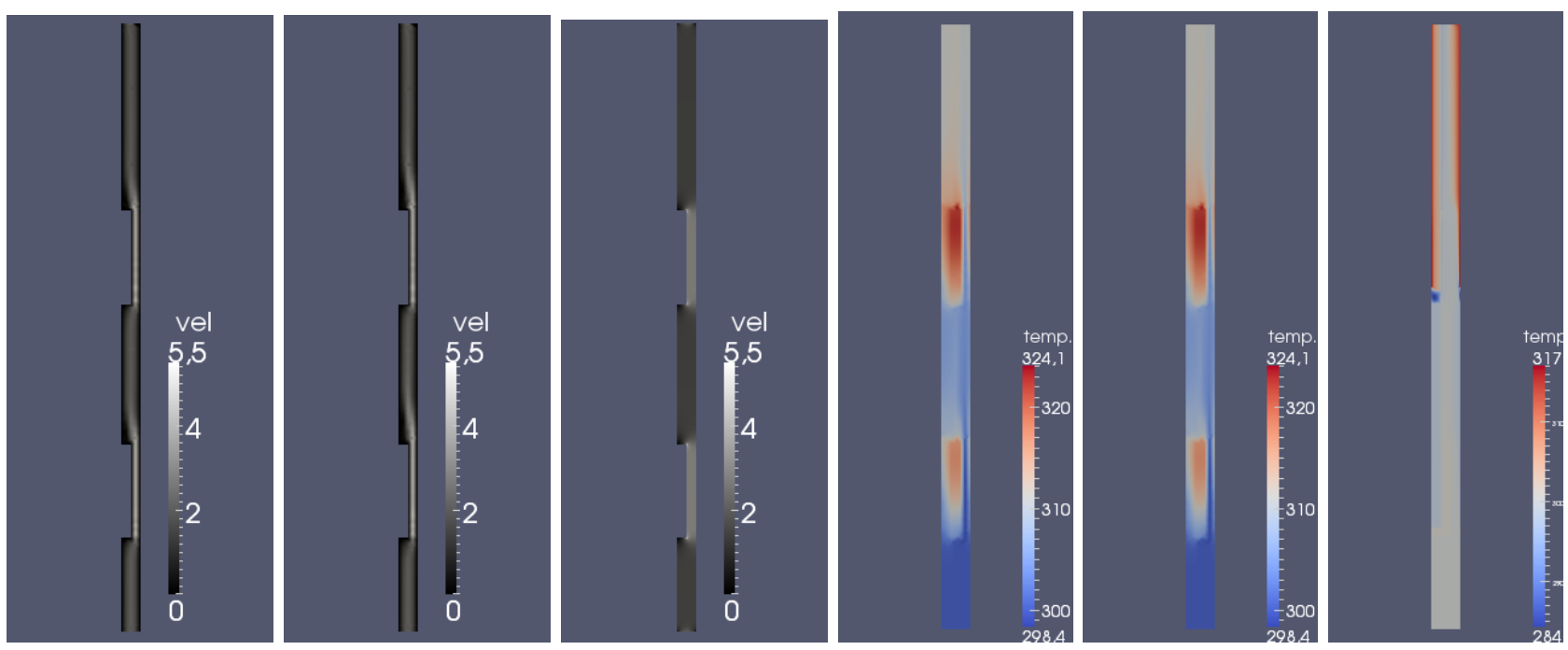

FiguRE 8. Left: velocity field, right: temperature field, for Boussinesq, NS and potential cases, in the international system of units.

decomposition in mechanical vibrations: the solution should be well represented by a small set of precomputed solutions (the reduced basis).

A wide variety of problems can be tackled by this method (see [8], [3]). We will present it in the context of the present study: the steady decoupled incompressible Navier-Stokes / heat model (3) applied to the electronic component.

The reduced basis method has been applied to a heat conduction problem by Sen (see [10]), and recently to the unsteady Boussinesq equations by Knezevic, Nguyen and Patera (see [7]). 
Consider that the fluid equations have been solved once for all, the RB method will be applied to the set of equations:

$$
\rho_{0} c_{p} \boldsymbol{u}_{\mathrm{NS}} \cdot \nabla T-\operatorname{div}(\kappa(x) \nabla T)=Q(x),
$$

where $\boldsymbol{u}_{\mathrm{NS}}$ is the result of the preliminary incompressible Navier-Stokes computation, and $Q(x)=q \mathbf{1}_{x \in \Omega_{\mathrm{IC}}}\left(\Omega_{\mathrm{IC}}\right.$ stands for integrated circuits and is defined in Figure 7$)$. $\boldsymbol{u}_{\mathrm{NS}}$ is extended by $\mathbf{0}$ in the integrated circuits and in the board.

Equation (12) can be rewritten as

$$
A_{\mu} T_{\mu}=f
$$

with $\mu$ a set of $n$ parameters (for instance $c_{p_{\text {IC }}}, \kappa_{\text {board }}$, etc...) in a $n$-dimensional subspace $\mathcal{D}$ of $\mathbb{R}^{n}$. Intervals of variation for the parameters are specified in section 3.3.3. Here, $A_{\mu}$ is the linear operator of the problem, $f$ the source term and $T_{\mu}$ represents the temperature, solution of equation (12) with the parameters set $\mu$.

Let us first check that the RB approach is reasonable in the present case, by selecting by hand a finite set of parameter values, computing the corresponding solutions $T_{i}$, selecting a problem-related scalar product $(T, \Theta)=\int_{\Omega} \nabla T \cdot \nabla \Theta$, and checking the variation of the eigenvalues of the matrix $M_{i j}=\left(T_{i}, T_{j}\right)$.

This is close to finding principal components of the energy operator (like modal basis). On Figure 9, we see

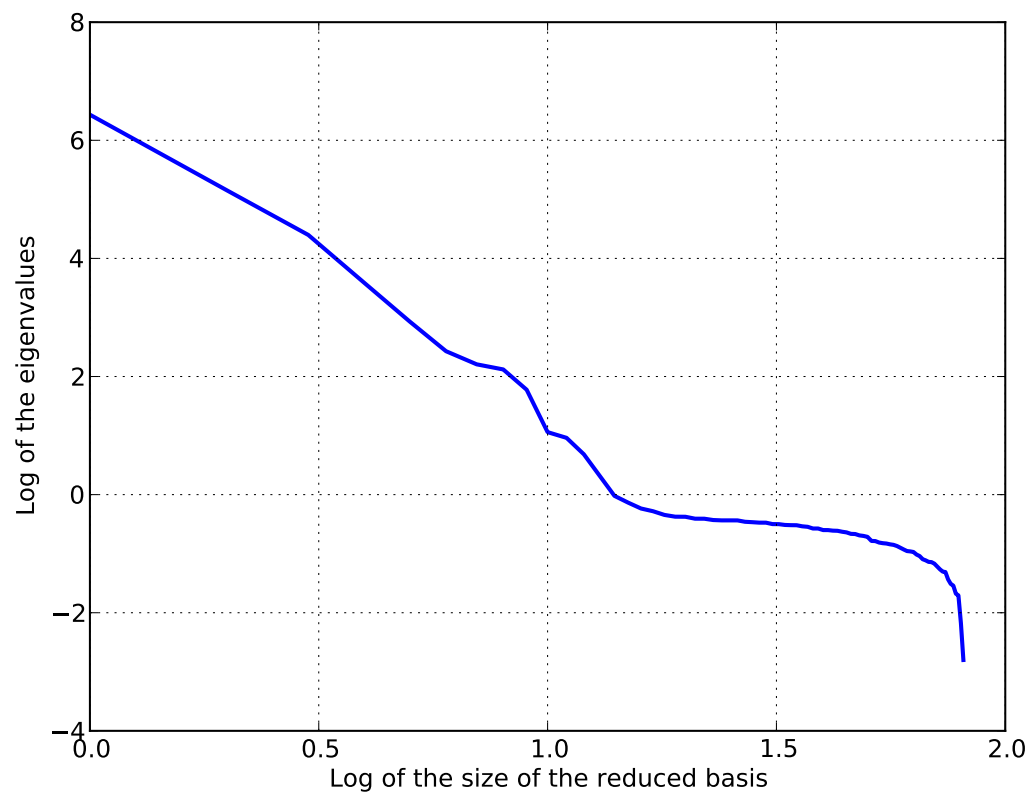

FiguRE 9. Eigenvalues of the Gram matrix $M$

that the eigenvalues of the Gram matrix $M$ of the problem lose 8 orders of magnitude with a reduced basis of size 81 chosen randomly, and the decreasing is exponential. This indicates that the solutions, for $\mu \in \mathcal{D}$, can be efficiently represented by a linear combination of a small number of functions. 
We are interested in the variational formulation of equation (13):

Find $T_{\mu} \in M_{h}$ such that $\forall \Theta \in M_{h}$

$$
a_{\mu}\left(T_{\mu}, \Theta\right)=l(\Theta)
$$

where $M_{h}$ is a finite dimensional subspace of $X:=H_{\mathrm{per}, 0}^{1}(\Omega)=\left\{T \in H^{1}(\Omega) \mid T_{\Gamma_{\mathrm{in}}}=0, T\right.$ periodic at $\left.\Gamma_{\text {per }}\right\}$, (we can make the Dirichlet condition homogeneous, considering a lifting of $\left.T_{\text {in }}\right)$. We use the Lagrange's $\mathbb{P}_{1}$ finite elements for this heat equation (see section 2.3). Since we have mixed boundary condition with a homogeneous Dirichlet condition, $\|\cdot\|_{H_{0}^{1}(\Omega)}$ is a norm on $H_{\text {per }, 0}^{1}(\Omega)$. The considered output is $s_{\mu}=l\left(T_{\mu}\right)=\left(f, T_{\mu}\right)_{H_{0}^{1}(\Omega)}=$ $q \int_{\Omega_{I C}} T_{\mu}$, a quantity proportional to the mean temperature in the integrated circuits. This specific output allows efficient mathematical analysis.

The RB consists in two steps:

- A computationally heavy offline stage: construct a low dimensional basis, which is a good basis for the high dimensional problem (12) for every parameter $\mu$ in $\mathcal{D}$,

- Fast online stages: solve light low dimensional problems.

The challenge of this approach is to guaranty that the approximate solution of equation (12) is a good one. This is possible thanks to the efficient computation of an a posteriori error estimate for our specific quantity of interest.

\subsection{Goal-oriented a posteriori error estimate : certified RB}

Consider $T_{\mu}^{\mathrm{FE}}$ the solution of (14) and $T_{\mu}^{\mathrm{RB}}$ the current RB approximation of (14) (the proper construction of $T_{\mu}^{\mathrm{RB}}$ is explained in section 3.3). These quantities verify $a_{\mu}\left(T_{\mu}^{\mathrm{FE}}, \Theta\right)=l(\Theta), \forall \Theta \in M_{h}$ and $a_{\mu}\left(T_{\mu}^{\mathrm{RB}}, \Theta\right)=$ $l(\Theta), \forall \Theta \in M_{\mathrm{RB}}$, where $M_{\mathrm{RB}}$ is the space spanned by the current reduced basis, and has much lower dimension than $M_{h}$.

The quantity of interest is $s_{\mu}^{\mathrm{FE}}$, which is computed using the expensive finite element solution, is approximated by $s_{\mu}^{\mathrm{RB}}$, which is computed using reduced basis approximation. We need an accurate and fast computable a posteriori error estimate $\Delta_{\mu}$ for the approximation error between the RB and the FE solution : $\left|s_{\mu}^{\mathrm{RB}}-s_{\mu}^{\mathrm{FE}}\right|$. By "fast", we mean that the evaluation of $\Delta_{\mu}$ should not require the computation of the FE solution.

The bilinear form $a_{\mu}$ in Equation (14) is coercive and non symmetric: given $\boldsymbol{u}_{\mathrm{NS}} \in V_{h}^{2}\left(\Omega_{\text {air }}\right)$ we seek $T \in M_{h}$ such that for all $\Theta \in M_{h}$ :

$$
a_{\mu}(T, \Theta)=\int_{\Omega} \rho_{0} c_{p}\left(\boldsymbol{u}_{\mathrm{NS}} \cdot \nabla \Theta\right) T+k \nabla \Theta \cdot \nabla T+\frac{1}{2} \int_{\Omega} \rho_{0} c_{p} \operatorname{div}\left(\boldsymbol{u}_{\mathrm{NS}}\right) T \Theta .
$$

where the last integral is the Temam term

The non-symmetry is induced by the convective term $\boldsymbol{u}_{\mathrm{NS}} \cdot \nabla T$. Recall that the precomputed velocity field $\boldsymbol{u}_{\mathrm{NS}}$ is incompressible and cancels on $\partial \Omega$ :

$$
\begin{aligned}
a_{\mu}(T, T) & =\int_{\Omega} \rho_{0} c_{p}\left(\boldsymbol{u}_{\mathrm{NS}} \cdot \nabla T\right) T+\int_{\Omega} k \nabla T \cdot \nabla T+\frac{1}{2} \int_{\Omega} \rho_{0} c_{p} \operatorname{div}\left(\boldsymbol{u}_{\mathrm{NS}}\right) T^{2} \\
& =-\frac{1}{2} \int_{\Omega} \rho_{0} c_{p} \operatorname{div}\left(\boldsymbol{u}_{\mathrm{NS}}\right) T^{2}+\int_{\Omega} k \nabla T \cdot \nabla T+\frac{1}{2} \int_{\Omega} \rho_{0} c_{p} \operatorname{div}\left(\boldsymbol{u}_{\mathrm{NS}}\right) T^{2} .
\end{aligned}
$$

Therefore, $a_{\mu}(T, T)=\int_{\Omega} \kappa_{\mu} \nabla T \cdot \nabla T \geq \min \left(\kappa_{\mu}\right)\|T\|_{H_{0}^{1}(\Omega)}^{2}$; the Temam term ensures coercivity for this bilinear form defined on $M_{h}^{2}\left(T \in \mathbb{P}_{1}\right.$, therefore $T^{2} \notin \mathbb{P}_{1}$ and $\left.\int_{\Omega} \operatorname{div}\left(\boldsymbol{u}_{\mathrm{NS}}\right) T^{2} \neq 0\right)$. This lower bound is quite pessimistic, especially when $\kappa_{\mu}$ varies a lot in the computational domain. For simplicity, we take this one in the present work. Methods exist to determine sharper constant for the discrete problem, based on solving eigenvalue problems (see [1] remark 16 p.114 for symmetric problems, see [6] for a presentation of the successive constraint method 
used in nonsymmetric problems).

We introduce the adjoint problem of (14):

Find $\Psi_{\mu}^{* \mathrm{FE}} \in M_{h}$ such that $\forall v \in M_{h}$

$$
a_{\mu}\left(v, \Psi_{\mu}^{* \mathrm{FE}}\right)=-l(v)
$$

We have to construct a reduced basis for this problem as well: define $\Psi_{\mu}^{* \mathrm{RB}}$ the current RB approximation of (16) (verifying $a_{\mu}\left(v, \Psi_{\mu}^{* \mathrm{RB}}\right)=-l(v), \forall v \in M_{\mathrm{RB}}^{*}$, the space spanned by the current reduced basis of (16)).

Consider the residual for the direct and the adjoint problems: for all $w, v \in M_{h}$,

$$
\left\{\begin{array}{l}
g_{\mu}(w, v)=a_{\mu}(w, v)-l(v) \\
g_{\mu}^{*}(w, v)=a_{\mu}(w, v)+l(w)
\end{array}\right.
$$

Make use of the version of Riesz-Fréchet representation theorem applied to continuous bilinear forms: there exists a unique application $G_{\mu}: M_{h} \longmapsto M_{h}$ such that $\forall w, v \in M_{h}, g_{\mu}(w, v)=\left(G_{\mu} w, v\right)_{H_{0}^{1}}$. Define $G_{\mu}^{*}$ in the same way: $\forall w, v \in M_{h}, g_{\mu}^{*}(w, v)=\left(w, G_{\mu}^{*} v\right)_{H_{0}^{1}}\left(\right.$ note that $G_{\mu} T_{\mu}^{\mathrm{FE}}=0$ and $\left.G_{\mu}^{*} \Psi_{\mu}^{* \mathrm{FE}}=0\right)$.

The output RB approximation of $s_{\mu}^{\mathrm{FE}}$ is computed as $s_{\mu}^{\mathrm{RB}, * \mathrm{RB}}=l\left(T_{\mu}^{\mathrm{RB}}\right)+g_{\mu}\left(T_{\mu}^{\mathrm{RB}}, \Psi_{\mu}^{* \mathrm{RB}}\right)$ (see [1] section 4-I-B-c). This specific output has been chosen to ensure the posteriori estimate given in the proposition 3.1. Note that the FE corresponding quantity is $l\left(T_{\mu}^{\mathrm{FE}}\right)+g_{\mu}\left(T_{\mu}^{\mathrm{FE}}, \Psi_{\mu}^{* \mathrm{FE}}\right)=l\left(T_{\mu}^{\mathrm{FE}}\right)=s_{\mu}^{\mathrm{FE}}$, so $g_{\mu}\left(T_{\mu}^{\mathrm{RB}}, \Psi_{\mu}^{* \mathrm{RB}}\right)$ only contains approximation errors introduced by the two reduced basis.

Make use of proposition 23 p.115 of Boyaval's PhD thesis [1] (see also [2] eq.22):

\section{Proposition 3.1.}

$$
\left|s_{\mu}^{\mathrm{RB}, * \mathrm{RB}}-s_{\mu}^{\mathrm{FE}}\right| \leq \Delta_{\mu}:=\frac{\left\|G_{\mu} T_{\mu}^{\mathrm{RB}}\right\|_{H_{0}^{1}(\Omega)}\left\|G_{\mu}^{*} \Psi_{\mu}^{* \mathrm{RB}}\right\|_{H_{0}^{1}(\Omega)}}{\alpha_{\mathrm{LB}, \mu}}
$$

where $\alpha_{\mathrm{LB}, \mu}$ is a computable lower bound for the coercivity constant $\alpha_{\mu}$ of $a_{\mu}(.,$. (recall that a bound for the continuous case is $\alpha_{\mathrm{LB}, \mu}=\min \left(\kappa_{\mu}\right)$ ).

Proof. Using elements of proof from Boyaval's PhD thesis (propositions 18 and 23):

$$
\left|s_{\mu}^{\mathrm{RB}, * \mathrm{RB}}-s_{\mu}^{\mathrm{FE}}\right|=\left|l\left(T_{\mu}^{\mathrm{FE}}-T_{\mu}^{\mathrm{RB}}\right)-g_{\mu}\left(T_{\mu}^{\mathrm{RB}}, \Psi_{\mu}^{* \mathrm{RB}}\right)\right| .
$$

Using (16), $T_{\mu}^{\mathrm{FE}}-T_{\mu}^{\mathrm{RB}} \in M_{h} \Longrightarrow l\left(T_{\mu}^{\mathrm{FE}}-T_{\mu}^{\mathrm{RB}}\right)=-a_{\mu}\left(T_{\mu}^{\mathrm{FE}}-T_{\mu}^{\mathrm{RB}}, \Psi_{\mu}^{* \mathrm{FE}}\right)$.

Using (14), $\Psi_{\mu}^{* \mathrm{FE}} \in M_{h} \Longrightarrow a_{\mu}\left(T_{\mu}^{\mathrm{EF}}, \Psi_{\mu}^{* \mathrm{FE}}\right)=l\left(\Psi_{\mu}^{* \mathrm{FE}}\right)$.

Then, $\left|s_{\mu}^{\mathrm{RB}, * \mathrm{RB}}-s_{\mu}^{\mathrm{FE}}\right|=\left|a_{\mu}\left(T_{\mu}^{\mathrm{RB}}, \Psi_{\mu}^{* \mathrm{FE}}\right)-l\left(\Psi_{\mu}^{* \mathrm{FE}}\right)-g_{\mu}\left(T_{\mu}^{\mathrm{RB}}, \Psi_{\mu}^{* \mathrm{RB}}\right)\right|=\left|g_{\mu}\left(T_{\mu}^{\mathrm{RB}}, \Psi_{\mu}^{* \mathrm{FE}}-\Psi_{\mu}^{* \mathrm{RB}}\right)\right|$, by definition (17).

Using Cauchy-Schwarz inequality, we can write:

$$
\left|s_{\mu}^{\mathrm{RB}, * \mathrm{RB}}-s_{\mu}^{\mathrm{FE}}\right| \leq\left\|G_{\mu} T_{\mu}^{\mathrm{RB}}\right\|_{H_{0}^{1}}\left\|\Psi_{\mu}^{* \mathrm{FE}}-\Psi_{\mu}^{* \mathrm{RB}}\right\|_{H_{0}^{1}}
$$


Then by coercivity, (16), (17) and the Cauchy-Schwarz inequality:

$$
\begin{aligned}
\left\|\Psi_{\mu}^{* \mathrm{FE}}-\Psi_{\mu}^{* \mathrm{RB}}\right\|_{H_{0}^{1}}^{2} & \leq \frac{\left|a_{\mu}\left(\Psi_{\mu}^{* \mathrm{FE}}-\Psi_{\mu}^{* \mathrm{RB}}, \Psi_{\mu}^{* \mathrm{FE}}-\Psi_{\mu}^{* \mathrm{RB}}\right)\right|}{\alpha_{\mathrm{LB}, \mu}} \\
& =\frac{\left|a_{\mu}\left(\Psi_{\mu}^{* \mathrm{FE}}-\Psi_{\mu}^{* \mathrm{RB}}, \Psi_{\mu}^{* \mathrm{RB}}\right)+l\left(\Psi_{\mu}^{* \mathrm{FE}}-\Psi_{\mu}^{* \mathrm{RB}}\right)\right|}{\alpha_{\mathrm{LB}, \mu}} \\
& =\frac{\left|g_{\mu}^{*}\left(\Psi_{\mu}^{* \mathrm{FE}}-\Psi_{\mu}^{* \mathrm{RB}}, \Psi_{\mu}^{* \mathrm{RB}}\right)\right|}{\alpha_{\mathrm{LB}, \mu}} \\
& \leq \frac{\left\|G_{\mu}^{*} \Psi_{\mu}^{* \mathrm{RB}}\right\|_{H_{0}^{1}}}{\alpha_{\mathrm{LB}, \mu}}\left\|\Psi_{\mu}^{* \mathrm{FE}}-\Psi_{\mu}^{* \mathrm{RB}}\right\|_{H_{0}^{1}} .
\end{aligned}
$$

Plugging this in (19), we get the inequality (3.1).

Remark 3.2. Note that the error estimate $\Delta_{\mu}$ does not require the evaluation of the FE solutions, enabling fast computation.

The inexpensive a posteriori error estimate (3.1) is useful to check rapidly if an online call approximates well the FE reference and to construct iteratively the reduced basis using a greedy algorithm (see section 3.3). This algorithm was proposed by Patera, Prud'homme, Rovas and Veroy in [9].

\subsection{Computation aspects and construction of the basis with a greedy algorithm}

\subsubsection{Offline stage: precomputation and greedy algorithm}

The certified RB method consists in iteratively construct a basis with solutions of the considered problem, computed at particular values of the parameters $\mu$.

Ideally, we would like to choose a tolerance $\epsilon$ for the error $\left|s_{\mu}^{\mathrm{RB}, * \mathrm{RB}}-s_{\mu}^{F E}\right|$ and make use of the error estimate $\Delta_{\mu}$ to construct a low dimensional basis where the error is guaranteed to be smaller than $\epsilon$ for all $\mu$ in the parameter space $\mathcal{D}$. Unfortunately, such a result does not exist. One can refer to recent work by Buffa, Maday, Patera, Prud'homme and Turinici [4] for theoretical results on greedy algorithms convergence performances.

In the present setting, we have an affine dependence of the operator on the parameters (thanks to equations (2)): for all $w, v \in M_{h}$,

$$
a_{\mu}(w, v)=a_{0}(w, v)+\sum_{i=1}^{n} \mu_{i} a_{i}(w, v)=\sum_{i=0}^{n} \mu_{i} a_{i}(w, v)
$$

In the same fashion, the residuals are written: for all $w, v \in M_{h}$,

$$
\left\{\begin{array}{l}
g_{\mu}(w, v)=-l(v)+\sum_{i=0}^{n} \mu_{i} a_{i}(w, v) \\
g_{\mu}^{*}(w, v)=l(w)+\sum_{i=0}^{n} \mu_{i} a_{i}(w, v)
\end{array}\right.
$$

where the $a_{i}$ do not depend on $\mu$.

Define now $G_{i}, G_{i}^{*}: \forall w, v \in M_{h}, \forall i=1, \ldots n,\left(G_{i} w, v\right)_{H_{0}^{1}}=a_{i}(w, v)$ and $\left(w, G_{i}^{*} v\right)_{H_{0}^{1}}=a_{i}^{*}(w, v)$. Take $g_{0}$ and $g_{0}^{*}$, solutions of $\left(g_{0}, v\right)_{H_{0}^{1}}=-l(v)$ and $\left(w, g_{0}^{*}\right)_{H_{0}^{1}}=l(w)$ (note that in this case, $g_{0}=-g_{0}^{*}$ ). 
In practice, we only need to compute $G_{i} w$ and $G_{i}^{*} v$ for given vectors $w, v \in M_{h}$. This is done by solving variational problems. Consider for instance $G_{i} w \in M_{h}$ :

Let $w \in M_{h}$. Find $u \in M_{h}$ such that $\forall v \in M_{h}$ :

$$
\int_{\Omega} \nabla u \cdot \nabla v=a_{i}(w, v)
$$

$w$ being fixed, $v \longmapsto a_{i}(w, v)$ is a continuous linear form, and $G_{i} w:=u$.

The other quantities defined above are computed in the same way. This will speed up the online stage since lots of quantities will be precalculated during the offline stage, letting to the online stage simple low dimension algebra operations (see section 3.3.2).

Take a finite set $\mathcal{D}_{\text {trial }} \subset \mathcal{D}$. The greedy algorithm reads as follow:

(i) Choose $\mu_{1} \in \mathcal{D}_{\text {trial }}$ randomly and remove it from $\mathcal{D}_{\text {trial }}$;

(ii) Compute $T_{\mu_{1}}^{\mathrm{FE}}$ and $\Psi_{\mu_{1}}^{* \mathrm{FE}}$, then set $V_{1}^{\mathrm{RB}}=\operatorname{Span}\left(T_{\mu_{1}}^{\mathrm{FE}}\right)$ and $V_{1}^{\mathrm{RB}}=\operatorname{Span}\left(\Psi_{\mu_{1}}^{* \mathrm{FE}}\right)$;

(iii) Compute $G_{i} T_{\mu_{1}}^{\mathrm{FE}}$ and $G_{i}^{*} \Psi_{\mu_{1}}^{* \mathrm{FE}}, \forall i \in[0, \ldots n]$, as well as $g_{0}$ and $g_{0}^{*}$;

(iv) Store $a_{i}\left(T_{\mu_{1}}^{\mathrm{FE}}, T_{\mu_{1}}^{\mathrm{FE}}\right),\left(G_{i} T_{\mu_{1}}^{\mathrm{FE}}, G_{i} T_{\mu_{1}}^{\mathrm{FE}}\right)_{H_{0}^{1}},\left(G_{i}^{*} \Psi_{\mu_{1}}^{* \mathrm{FE}}, G_{i}^{*} \Psi_{\mu_{1}}^{* \mathrm{FE}}\right)_{H_{0}^{1}},\left(g_{0}, G_{i} T_{\mu_{1}}^{\mathrm{FE}}\right)_{H_{0}^{1}}$ and $\left(g_{0}^{*}, G_{i}^{*} \Psi_{\mu_{1}}^{* \mathrm{FE}}\right)_{H_{0}^{1}}$ $\forall i \in[0, \ldots n]$, as well as $\left(g_{0}, g_{0}\right)_{H_{0}^{1}}$ and $\left(g_{0}^{*}, g_{0}^{*}\right)_{H_{0}^{1}}$;

(v) While $\max \left\{\Delta_{\mu_{i}}, \mu_{i} \in \mathcal{D}_{\text {trial }}\right\} \geq \epsilon$

- Solve $\mu_{N}=\operatorname{argmax}\left\{\Delta_{\mu_{\mathrm{i}}}, \mu_{\mathrm{i}} \in \mathcal{D}_{\text {trial }}\right\}$ and remove it from $\mathcal{D}_{\text {trial }}$;

- Compute $T_{\mu_{N}}^{\mathrm{FE}}$ and $\Psi_{\mu_{N}}^{* \mathrm{FE}}$, then set $V_{N}^{\mathrm{RB}}=\operatorname{Span}\left(T_{\mu_{1}}^{\mathrm{FE}}, \ldots T_{\mu_{N}}^{\mathrm{FE}}\right)$ and $V_{N}^{\mathrm{RB}}=\operatorname{Span}\left(\Psi_{\mu_{1}}^{* \mathrm{FE}}, \ldots \Psi_{\mu_{N}}^{* \mathrm{FE}}\right)$;

- Compute $G_{i} T_{\mu_{N}}^{\mathrm{FE}}$ and $G_{i}^{*} \Psi_{\mu_{N}}^{* \mathrm{FE}}, \forall 0 \in[1, \ldots n]$;

- Store $a_{i}\left(T_{\mu_{k}}^{\mathrm{FE}}, T_{\mu_{N}}^{\mathrm{FE}}\right),\left(G_{i} T_{\mu_{k}}^{\mathrm{FE}}, G_{j} T_{\mu_{N}}^{\mathrm{FE}}\right)_{H_{0}^{1}}$ and $\left(G_{i}^{*} \Psi_{\mu_{k}}^{* \mathrm{FE}}, G_{j}^{*} \Psi_{\mu_{N}}^{* \mathrm{FE}}\right)_{H_{0}^{1}} \forall i, j \in[0, \ldots n], \forall k \in[1, \ldots N]$, as well as $\left(g_{0}, G_{i} T_{\mu_{N}}^{\mathrm{FE}}\right)_{H_{0}^{1}}$ and $\left(g_{0}^{*}, G_{i}^{*} \Psi_{\mu_{N}}^{* \mathrm{FE}}\right)_{H_{0}^{1}} \forall i \in[0, \ldots n]$;

Remark: since the problem is not symmetric, we also have to store $a_{i}\left(T_{\mu_{N}}^{\mathrm{FE}}, T_{\mu_{k}}^{\mathrm{FE}}\right),\left(G_{i} T_{\mu_{N}}^{\mathrm{FE}}, G_{j} T_{\mu_{k}}^{\mathrm{FE}}\right)_{H_{0}^{1}}$ and $\left(G_{i}^{*} \Psi_{\mu_{N}}^{* \mathrm{FE}}, G_{j}^{*} \Psi_{\mu_{k}}^{* \mathrm{FE}}\right)_{H_{0}^{1}} \forall i, j \in[0, \ldots n], \forall k \in[1, \ldots N-1]$;

- $N=N+1$

Note that we did not explain how to chose the finite set $\mathcal{D}_{\text {trial }}$. In our case, we simply discretized each direction of $\mathcal{D}$ with a constant step: see Figure 10. The efficient evaluation of $\Delta_{\mu}$ is explained in the next section 3.3.2.
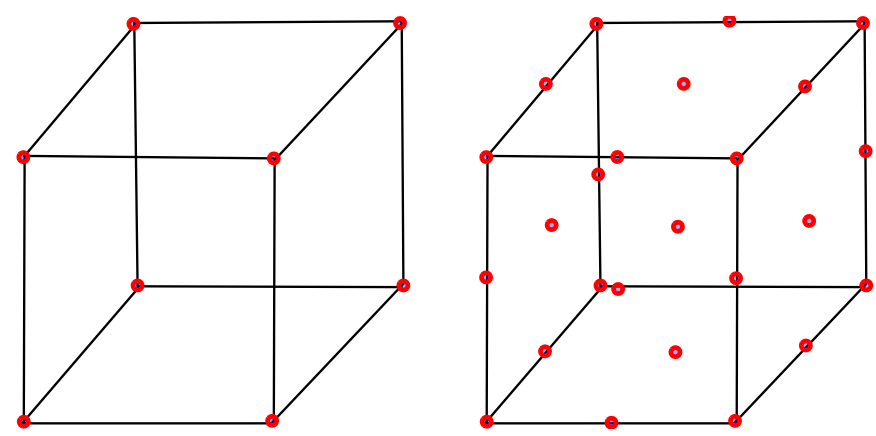

FiguRE 10. Determination of $\mathcal{D}_{\text {trial }}$ in the case of tridimensional parameter space. Left: two values per direction, right: three values per direction 


\subsubsection{Online stage}

Consider that a basis $(b)_{i}$ of size $d$ has been constructed (and a basis $\left(b^{*}\right)_{i}$ for the adjoint problem), and that we want to efficiently compute the temperature using the RB for a given parameter.

An online call consists in solving the following Galerkin problem: Find $T_{\mu}^{\mathrm{RB}} \in M^{\mathrm{RB}}:=\operatorname{Vect}\left(b_{1}, b_{2}, \ldots b_{d}\right)$ such that $\forall v \in M^{\mathrm{RB}}$

$$
a_{\mu}\left(T_{\mu}^{\mathrm{RB}}, v\right)=l(v)
$$

Write $T^{\mathrm{RB}}=\sum_{i=1}^{d} \alpha_{i} b_{i}$, the Galerkin problem becomes: Find $\left(\alpha_{1}, \alpha_{2}, \ldots \alpha_{d}\right)$ such that $\forall j=1, \ldots d$

$$
\sum_{i=1}^{d} \alpha_{i} a_{\mu}\left(b_{i}, b_{j}\right)=l\left(b_{j}\right)
$$

We have $A_{\mu}:=\sum_{k=1}^{d} \mu_{k}\left(a_{k}\left(b_{i}, b_{j}\right)\right)_{i j}$, where all $a_{k}\left(b_{i}, b_{j}\right)$ have been computed and stored during the offline stage.

Defining the vectors $(\alpha)_{j}=\alpha_{j}$, and $(F)_{j}=l\left(b_{j}\right)$, one then just has to solve the $d$-dimensional linear system

$$
A_{\mu} \alpha=F
$$

and then compute the $\mathrm{RB}$ solution $u_{\mu}^{\mathrm{RB}}=\sum_{i=1}^{d} \alpha_{i} b_{i}$. Note that the $\alpha_{i}$ contain the dependence of the solution on $\mu$.

In practice, when the size of the reduced basis increases, the matrix $A_{\mu}$ may be close to singular. One can improve this by orthonormalizing the basis using modified or simple Gram-Schmidt (see [1], p.109).

Then, an online call should also contain an evaluation of the a posteriori error estimate $\Delta_{\mu}$, and if it is larger than $\epsilon$, one can compute the FE solution and enrich the basis with this function. This way, the error estimate is guaranteed, but we may need to compute the expensive solution sometimes.

We also use precomputed quantities to get an evaluation of $\Delta_{\mu}$ in a complexity independent of the FE problem size.

$$
\begin{aligned}
\left\|G_{\mu} T_{\mu}^{\mathrm{RB}}\right\|_{H_{0}^{1}}^{2} & =\left(G_{\mu} T_{\mu}^{\mathrm{RB}}, G_{\mu} T_{\mu}^{\mathrm{RB}}\right)_{H_{0}^{1}} \\
& =\left(g_{0}+\sum_{i}^{n} \mu_{i} G_{i} T_{\mu}^{\mathrm{RB}}, g_{0}+\sum_{j}^{n} \mu_{i} G_{j} T_{\mu}^{\mathrm{RB}}\right)_{H_{0}^{1}} \\
& =\left(g_{0}, g_{0}\right)_{H_{0}^{1}}+2 \sum_{i}^{n} \sum_{k}^{d} \mu_{i} \alpha_{k}\left(g_{0}, G_{i} b_{k}\right)_{H_{0}^{1}}+\sum_{i, j=1}^{n} \sum_{k, l=1}^{d} \mu_{i} \mu_{j} \alpha_{k} \alpha_{l}\left(G_{i} b_{k}, G_{j} b_{l}\right)_{H_{0}^{1}}
\end{aligned}
$$

using affine dependence

using RB decomposition

where all the scalar products involved have been precomputed and stored during the offline stage. Therefore

$$
\Delta_{\mu}=\frac{\left\|G_{\mu} T_{\mu}^{\mathrm{RB}}\right\|_{H_{0}^{1}(\Omega)}\left\|G_{\mu}^{*} \Psi_{\mu}^{* \mathrm{RB}}\right\|_{H_{0}^{1}(\Omega)}}{\alpha_{\mathrm{LB}, \mu}}
$$


is of complexity $O\left(n^{2} d^{2}\right)$, which corresponds to a fast computation compared to the FE problem. The faster the estimator is computed, the larger $\mathcal{D}_{\text {trial }}$ we can explore in the greedy algorithm for a given computation time.

\subsubsection{Numerical results}

The greedy algorithm has been carried out to compute 10 basis functions, with a parameter set of 20,000 points. The finite elements problem has 9012 degrees of freedom.

We took as parameters: $\kappa_{\mathrm{IC}}, \kappa_{\text {board }}, \kappa_{\text {air }}$ the integrated circuit, board and air heat conductivity and $c_{p_{\text {air }}}$ the air thermal capacity, varying in the following intervals (international system of units):

\begin{tabular}{|c|c|c|c|c|}
\hline & $\kappa_{\text {IC }}$ & $\kappa_{\text {board }}$ & $\kappa_{\text {air }}$ & $c_{p_{\text {air }}}$ \\
\hline $\min$ & 0.5 & 0.06 & 0.028 & 1080 \\
\hline $\max$ & 5 & 0.6 & 0.032 & 1120 \\
\hline
\end{tabular}

We first check that the growth of the basis reduces projection errors (see Figure 11):

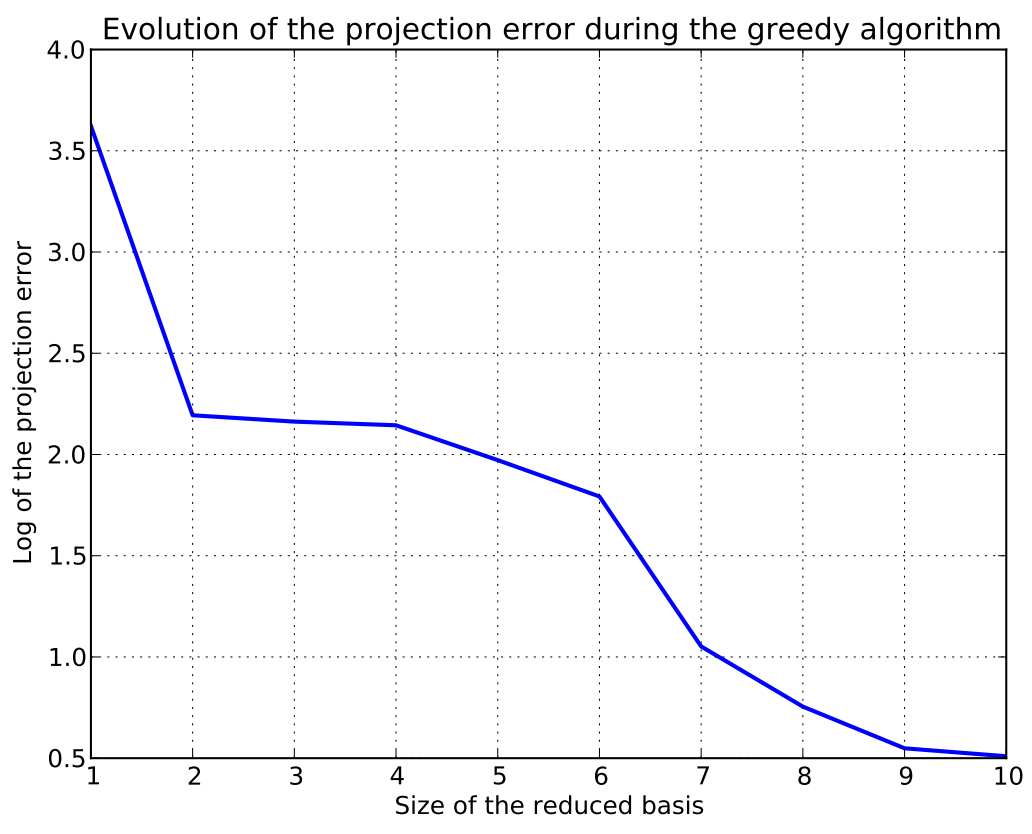

Figure 11. Evolution of $\left\|T_{\hat{\mu}}^{\mathrm{FE}}-T_{\hat{\mu}}^{\mathrm{RB}}\right\|_{H_{0}^{1}}$ for $\hat{\mu} \in \mathcal{D}$ taken randomly with the size of the reduced basis

Consider now the evolution of the maximum error estimate $\Delta_{\mu}$ for $\mu \in \mathcal{D}_{\text {trial }}$ and the corresponding value of the error $\left|s_{\mu}^{\mathrm{RB}, * \mathrm{RB}}-s_{\mu}^{\mathrm{FE}}\right|$ (see Figure 12). The error estimate and the error are not strictly decreasing. Maybe this is due to the fact that the RB is certified for a scalar quantity different from the projection error represented in Figure 11. Recall also that we did not compute a satisfying lower bound for the coercivity constant of the bilinear form $a_{\mu}$. The error estimate for a random parameter is not strictly decreasing with the size of the basis, but globally tends to zero. Remark that an error of $8 \times 10^{-5}$ correspond to a mean temperature error of $1 \mathrm{~K}$ 
over the integrated circuits.

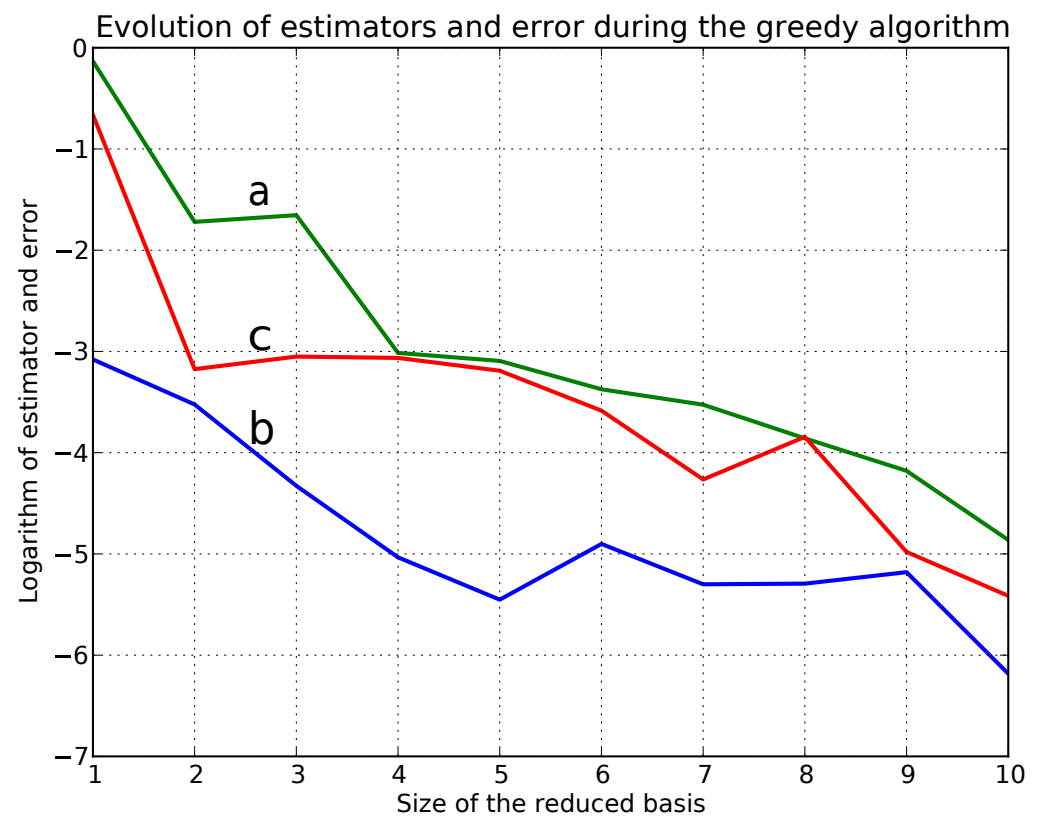

FiguRE 12. Evolution of the maximum error estimate $\Delta_{\mu}$ for $\mu \in \mathcal{D}_{\text {trial }}$ (a), the corresponding value of the error $\left|s_{\mu}^{\mathrm{RB}, * \mathrm{RB}}-s_{\mu}^{\mathrm{FE}}\right|$ (b) and the error estimate $\Delta_{\hat{\mu}}$ for $\hat{\mu} \in \mathcal{D}$ taken randomly (c)

Once the basis constructed, we tried an online call taking a random parameter in $\mathcal{D}$ (see Figure 13):

- A posteriori error estimate : $7.24 \times 10^{-6}$

- Error : $1.20 \times 10^{-6}$

- Integrated circuits temperature RB: $40.99 \mathrm{~K}$; direct computation: $40.96 \mathrm{~K}$ relative difference $=0.074 \%$

Duration of the different stages:

- offline with greedy: $8 \min 37 \mathrm{~s}$

- one finite element resolution: $0.28 \mathrm{~s}$

- one online call: $1.4 \times 10^{-6} \mathrm{~S}$

\section{CONCLUSION}

We developed different models for solving the velocity and the temperature of the air under different conditions. The full Boussinesq model yields satisfactory results in the cases we considered, whereas decoupled incompressible Navier-Stokes / heat was satisfactory for the electronic component case (forced convection in a pipe).

We then tested a certified RB method for the heat problem with non homogeneous convection. Our code makes use of an a posteriori error estimate to build a basis iteratively using a greedy algorithm. The affine parametric assumption allows to precompute many terms, and online calls are reduced to add and inverse low 

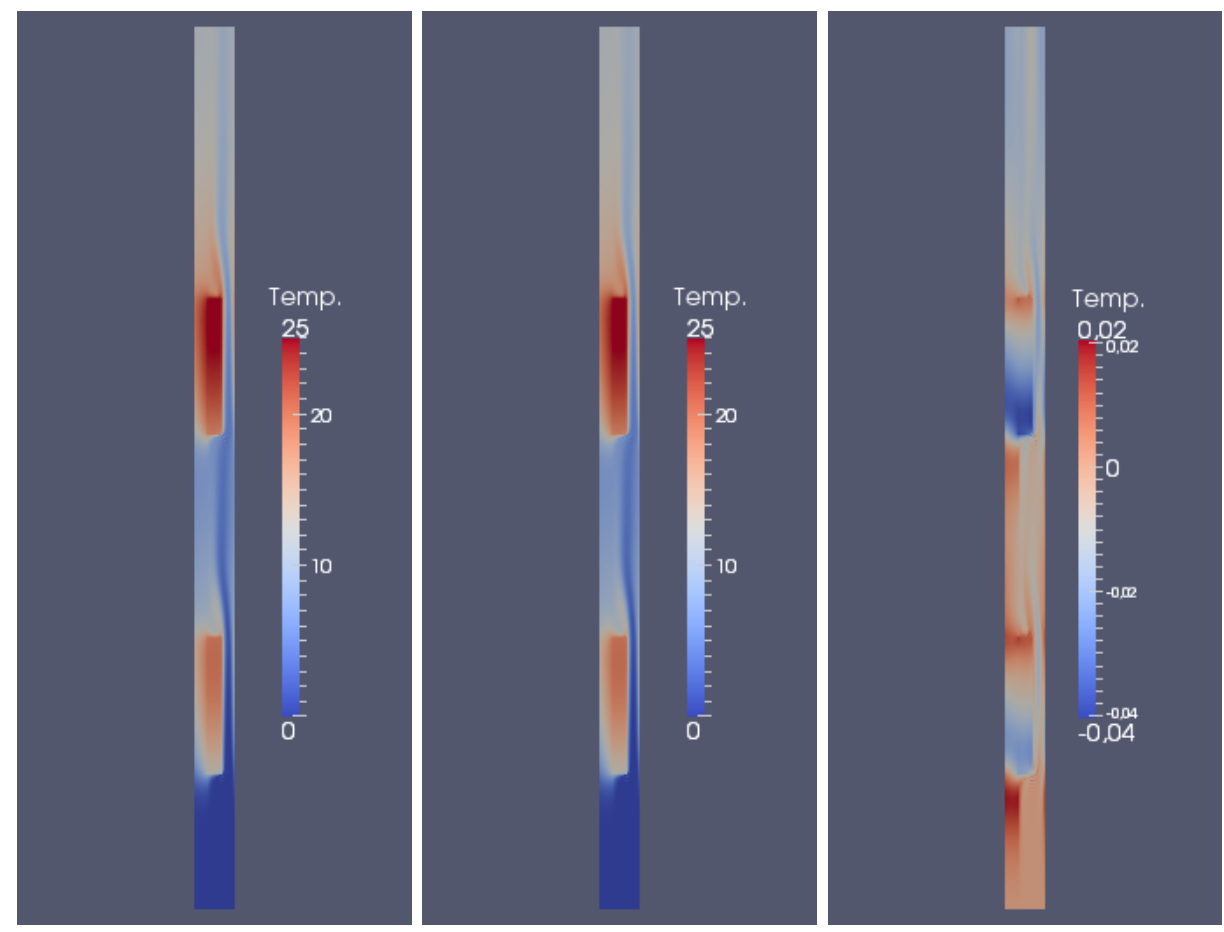

FiguRE 13. Temperature maps for the electronic component case. Left: RB online call $\boldsymbol{u}_{\mathrm{RB}}$, center: FE calculation $\boldsymbol{u}_{\mathrm{FE}}$, right: difference $\boldsymbol{u}_{\mathrm{FE}}-\boldsymbol{u}_{\mathrm{RB}}$

dimensional matrices. This enables us to drastically speed up the resolution in the electric component, with a physically satisfying model, and controlled approximation errors.

\section{ACKNOWLEDGMENTS}

This work was financially supported by EADS IW. The authors wish to thank the CEMRACS'11 organizers and Sébastien Boyaval, Albert Cohen, Alexandre Ern, Benoit Fabrèges and Tony Lelièvre for fruitful discussions.

\section{REFERENCES}

[1] S. Boyaval. Mathematical modelling and numerical simulation in materials science. PhD thesis, Université Paris-Est, Juin 2009.

[2] S. Boyaval, C. Le Bris, T. Lelièvre, Y. Maday, N.C. Nguyen, and A.T. Patera. Reduced basis techniques for stochastic problems. Archives of Computational Methods in Engineering, 17(4), 2010.

[3] S. Boyaval, C. Le Bris, Y. Maday, N.C. Nguyen, and A.T. Patera. A reduced basis approach for variational problems with stochastic parameters: Application to heat conduction with variable robin coefficient. Computer Methods in Applied Mechanics and Engineering, 198(41-44):3187 - 3206, 2009.

[4] A. Buffa, Y. Maday, A.T. Patera, C. Prud'homme, and G. Turinici. A priori convergence of the greedy algorithm for the parametrized reduced basis method. ESAIM: Mathematical Modelling and Numerical Analysis, 46(3):595-603, 2012.

[5] F. Hecht, J. Morice, and O. Pironneau. freeFEM++, www.freefem.org/.

[6] D.B.P. Huynh, A. T. Patera, G. Rozza, and S. Sen. A successive constraint linear optimization method for lower bounds of parametric coercivity and inf-sup stability constants. Comptes Rendus Mathematique, 345(8):473 - 478, 2007.

[7] D.J. Knezevic, N.C. Nguyen, and A.T. Patera. Reduced Basis Approximation and A Posteriori Error Estimation for the Parametrized Unsteady Boussinesq Equations. Mathematical Models \& Methods in applied sciences, 21(7):1415-1442, JUL 2011. 
[8] L. Machiels, Y. Maday, A.T. Patera, C. Prud'homme, D.V. Rovas, G. Turinici, and K. Veroy. Reliable real-time solution of parametrized partial differential equations: Reduced-basis output bound methods. Journal of Fluids Engineering, 124(70), 2002.

[9] A.T. Patera, D.V. Rovas C. Prud' homme, and K. Veroy. A posteriori error bounds for reduced-basis approximation of parametrized noncoercive and nonlinear elliptic partial differential equations. Proceedings of the 16th AIAA Computational Fluid Dynamics Conference, 2003.

[10] S. Sen. Reduced-basis approximation and a posteriori error estimation for many-parameter heat conduction problems. Numerical Heat Transfer, Part B: Fundamentals, 54(5):369-389, 2008. 